\title{
Asymptomatic Brain Lesions on Cranial Magnetic Resonance Imaging in Inflammatory Bowel Disease
}

\author{
Can Dolapcioglu*, Yuksel Guleryuzlu ${ }^{\dagger}$, Oya Uygur-Bayramicli ${ }^{\ddagger}$, Emel Ahishali*, and Resat Dabak $^{\S}$ \\ Departments of *Gastroenterology and ${ }^{\dagger}$ Internal Medicine, Dr. Lutfi Kirdar Kartal Research and Training Hospital, ${ }^{\ddagger}$ Department of \\ Gastroenterology, Maltepe University School of Medicine, and ${ }^{\varepsilon}$ Department of Family Medicine, Dr. Lutfi Kirdar Kartal Research and Training \\ Hospital, Istanbul, Turkey
}

Background/Aims: This study aimed to examine the frequency and type of asymptomatic neurological involvement in inflammatory bowel disease (IBD) using cranial magnetic resonance imaging (MRI). Methods: Fifty-one IBD patients with no known neurological diseases or symptoms and 30 controls with unspecified headaches without neurological origins were included. Patients and controls underwent cranial MRI assessments for white matter lesions, sinusitis, otitismastoiditis, and other brain parenchymal findings. Results: The frequencies of white matter lesions, other brainstem parenchymal lesions, and otitis-mastoiditis were similar in IBD patients and controls ( $p>0.05$ ), whereas sinusitis was significantly more frequent in IBD patients $(56.9 \%$ vs $33.3 \%$, $p=0.041$ ). However, among those subjects with white matter lesions, the number of such lesions was significantly higher in IBD patients compared to controls $(12.75 \pm 9.78$ vs $3.20 \pm 2.90, p<0.05)$. The incidence of examined pathologies did not differ significantly with disease activity ( $p>0.05$ for all). Conclusions: The incidence of white matter lesions seemed to be similar in IBD patients and normal healthy individuals, and the lesions detected did not pose any clinical significance. However, long-term clinical follow-up of the lesions is warranted. (Gut Liver 2013;7:169-174)

Key Words: Inflammatory bowel disease; Asymptomatic brain lesion; Magnetic resonance imaging; White matter lesions

\section{INTRODUCTION}

Although inflammatory bowel disease (IBD) is primarily a disease of large and small intestines, it is regarded as a systemic condition due to its effects in other organs and tissues. Extraintestinal signs of IBD generally involve bones, joints, skin, eye, or vasculature, and they are referred to as extraintestinal manifestations (EIM) of IBD. ${ }^{1}$ EIM may or may not show correlation with the disease activity. Prerequisites for the definition of EIM include a documented diagnosis of IBD; exclusion of side effects of steroids, 5-ASA derivatives, or immunosuppressant medications; and absence of other conditions such as Behçet's disease, vasculitides, and infections by such as Yersinia or Campylobacter that may cause systemic signs. Reported incidences of EIM in patients with IBD range between $6 \%$ and $47 \%{ }^{1}$

Neurological signs and complications that cannot be attributed to a primary neurological disorder are also classified among EIM of IBD. ${ }^{2}$ Despite the wide variation in the reported incidence of neurological signs (0.2\% to 35.7\%), the figures are low in most of the studies. ${ }^{2}$ Neurological complications of IBD have usually been presented as case reports with ill-defined pathogenesis and scarce information on their incidence. Although a variety of neurological signs and conditions in association with IBD such as peripheral nerve involvement, thromboembolic complications, myopathy, myelopathy, convulsions, cerebrovascular disease, major depression, focal central nervous system (CNS) disorders, demyelinating disease, confusion, meningitis, and syncope have been reported in several studies, ${ }^{2-4}$ the actual incidence of asymptomatic neurological involvement is unknown.

Scheid and Teich ${ }^{5}$ proposed the following three possible mechanisms for the peripheral and CNS involvement in ulcerative colitis (UC): cerebrovascular conditions due to thromboembolic events; systemic and cerebral vasculitis; and neuropathy and cerebral demyelination due to immune-related mechanisms. White matter lesion on the other hand is a frequent finding in

\footnotetext{
Correspondence to: Can Dolapcioglu

Department of Gastroenterology, Dr. Lutfi Kirdar Kartal Research and Training Hospital, Altunizade Mah. Piskinler Sok. Aykent Sitesi, B Blok Daire 13, Uskudar, Istanbul 34662, Turkey

Tel: +90-532-261-3919, Fax: +90-216-418-8752, E-mail: candolapci@hotmail.com Received on December 22, 2011. Revised on May 16, 2012. Accepted on June 15, 2012 pISSN 1976-2283 eISSN 2005-1212 http://dx.doi.org/10.5009/gnl.2013.7.2.169

(a) This is an Open Access article distributed under the terms of the Creative Commons Attribution Non-Commercial License (http://creativecommons.org/licenses/by-nc/3.0) which permits unrestricted non-commercial use, distribution, and reproduction in any medium, provided the original work is properly cited.
} 
patients with IBD on magnetic resonance imaging (MRI), and the development of these lesions have been attributed to ischemic mechanisms (atherosclerotic or vasculitic) or demyelination. ${ }^{6-8}$ Thus, early identification of these lesions may be clinically helpful as an early indication of neurological involvement, since they may represent another EIM of the disease. ${ }^{8}$

The aim of this study was to evaluate the frequency and type of asymptomatic brain lesions and its correlation with disease activity in IBD patients using cranial MRI.

\section{MATERIALS AND METHODS}

\section{Patients}

This cross-sectional study was conducted at the Department of Gastroenterology, Dr. Lutfi Kirdar Kartal Research and Training Hospital between April 2006 and October 2007 with the participation of 51 IBD patients with no known neurological disease and symptoms. Patients had either Crohn's disease or UC, none of them had indeterminate colitis. Neurological symptom is defined as subjective experience in the form of a sensation or change in bodily function that is potentially associated with neurological system. Patients with a prior diagnosis of neurological disorders, systemic, metabolic, and nutritional diseases, and those with drug related neurological complications were excluded. A total of 30 subjects with no known disease and attending to neurology outpatient clinic with a complaint of unspecified headache without neurological origin comprised the control group. The study protocol was approved by institutional ethics committee and the study was conducted in accordance with the Declaration of Helsinki. All subjects provided informed consent prior to study entry.

The diagnoses of UC and Crohn's disease were based on clinical, biochemical, endoscopic, histopathological, and radiological criteria and the disease activity was assessed using clinical activity scoring systems. Accordingly, Truelove-Witts criteria ${ }^{9}$ were used to classify UC patients in three activity groups: mild, moderate, and severe. Patients with Crohn's disease were scored according to eight different criteria using Crohn's disease activity index as follows: quiescent disease, below 150; moderate severity, 150 to 450 ; severe disease, greater than $450 .^{10}$

\section{MRI}

MRI in IBD and control patients was performed with a 1.5 Tesla superconductive magnet using the following techniques: axial fast spin echo (FSE) T2 (repetition time [TR], 3,870; echo delay time [TE], 111; field of view [FOV], 190; matrix, 340×512; cross-sectional thickness, $5 \mathrm{~mm}$; gap, $1 \mathrm{~mm}$ ), sagittal FSE T1 (TR, 582; TE, 10; FOV, 236; matrix, 192×256; cross-sectional thickness, $5 \mathrm{~mm}$; gap, $1 \mathrm{~mm}$ ), axial FSE T1 (TR, 570; TE, 15; FOV, 181; matrix, 340×512; cross-sectional thickness, $5 \mathrm{~mm}$; gap, $1 \mathrm{~mm}$ ), and coronal FLAIR (TR, 6,720; TE, 80; T1, 2,000; FOV, 186; matrix, 306×512; cross-sectional thickness, $5 \mathrm{~mm}$; gap, 1 $\mathrm{mm}$ ). The MRI assessments including those for white matter lesions, sinusitis, otitis-mastoiditis, and other brain parenchymal findings of the patients and controls were performed by a radiologist blinded to the groups. Subjects with pathological findings on MRI (white matter lesions or other parenchymal brain lesions) were examined by a neurologist blinded to the diagnosis.

\section{Statistical analyses}

Statistical analyses were performed using SPSS version 10.0 for Windows (SPSS Inc., Chicago, IL, USA). In addition to descriptive statistical methods (mean, standard deviation), MannWhitney U test was used for between-group comparisons of quantitative data. For the comparison of qualitative data, chisquare, and Fisher's exact test were used. Results were expressed at a significance level of $\mathrm{p}<0.05$.

\section{RESULTS}

\section{Patients}

Fifty-one IBD patients (29 male, 22 female; mean age, $42.92 \pm 14.97$ years; range, 16 to 73 years) and 30 controls (16 male, 14 male; mean age, $43.83 \pm 10.35$ years; range, 23 to 60 years) were included in the study. In IBD group, 37 subjects had UC (25 male, 12 female; mean age, 44.08 \pm 15.33 years; range, 16 to 73 years), and 14 had Crohn's disease (4 male, 10 female; mean age, $39.85 \pm 15.07$ years; range, 20 to 65 years). There were no significant differences between the groups with regard to age, BMI, and gender distribution ( $p>0.05$ ) as well as for the number of patients with hypertension, diabetes, dyslipidemia, and cigarette smoking ( $>0.05$ ) (Table 1).

Of the 37 patients with UC, 35.1\%, 24.3\%, and 40\% had pancolitis, left-sided disease, and distal disease (proctitis), respectively. In patients with Crohn's disease, 28.6\% and 42.9\% had

Table 1. Comparison of the Patients and Controls with Regard to Baseline Characteristics

\begin{tabular}{lccc}
\hline $\begin{array}{l}\text { Demographical } \\
\text { characteristics }\end{array}$ & IBD $(\mathrm{n}=51)$ & Control $(\mathrm{n}=30)$ & p-value \\
\hline Age, yr & $42.92 \pm 14.97$ & $43.83 \pm 10.35$ & $0.494^{*}$ \\
Male gender & $29(56.9)$ & $16(53.3)$ & $0.758^{\dagger}$ \\
BMI, kg/m & $25.21 \pm 12.34$ & $26.47 \pm 11.43$ & $0.412^{*}$ \\
Smoking & $7(13.7)$ & $4(13.3)$ & $0.960^{\dagger}$ \\
Diabetes & $4(7.8)$ & 0 & $0.291^{\dagger}$ \\
Hypertension & $8(15.7)$ & $2(6.7)$ & $0.233^{\dagger}$ \\
Dyslipidemia $^{\ddagger}$ & $5(9.8)$ & $5(16.7)$ & $0.365^{\dagger}$ \\
\hline
\end{tabular}

Data are presented as mean \pm SD or number $(\%)$. IBD, inflammatory bowel disease; BMI, body mass index.

${ }^{*}$ Mann Whitney U test; ${ }^{\dagger}$ Chi-square test or Fisher's exact test, where appropriate; ${ }^{\ddagger}$ Dyslipidemia was defined as the presence of at least one of the following: low density lipoprotein cholesterol $>160 \mathrm{mg} / \mathrm{dL}$, total cholesterol $>240 \mathrm{mg} / \mathrm{dL}$, or triglycerides $>200 \mathrm{mg} / \mathrm{dL}$. 


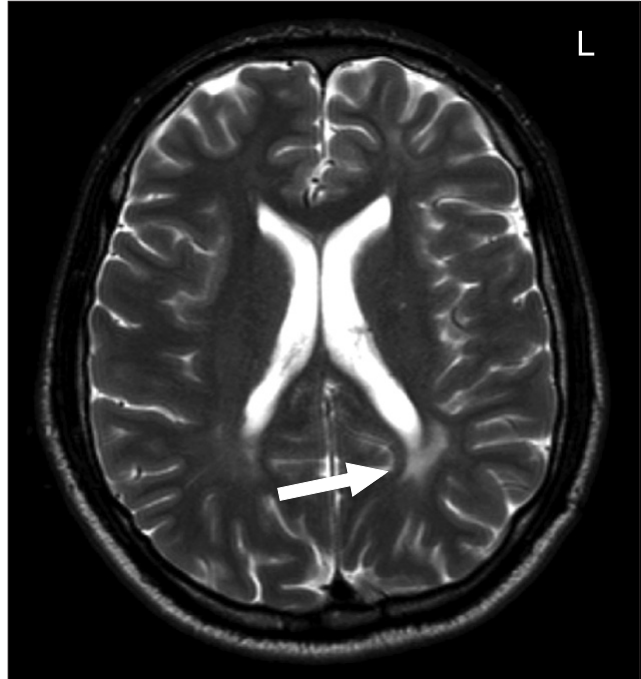

Fig. 1. T2-weighted magnetic resonance image of a 31-year-old female patient with ulcerative colitis shows a large white matter lesion on the left side (arrow).

L, left.

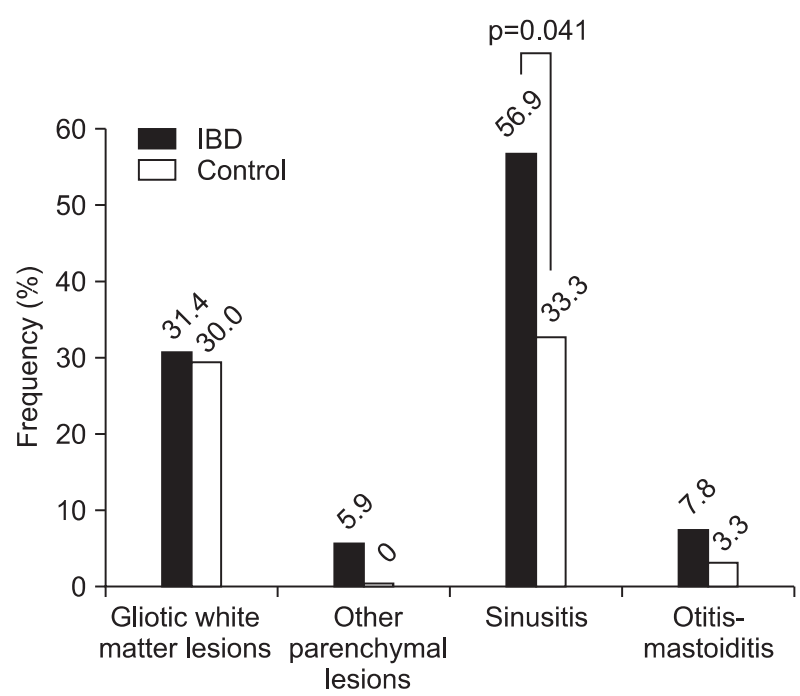

Fig. 2. Frequencies of pathological magnetic resonance imaging findings among inflammatory bowel disease (IBD) patients and controls. For the comparisons, the chi-square test or Fisher's exact test was used as appropriate.

Table 2. Comparison of Ulcerative Colitis and Crohn's Disease Patients with Regard to Cranial Magnetic Resonance Imaging Findings

\begin{tabular}{lccc}
\hline & Ulcerative colitis $(\mathrm{n}=37)$ & Crohn’s disease $(\mathrm{n}=14)$ & $\mathrm{p}$-value* \\
\hline White matter lesions & $14(37.8)$ & $2(14.3)$ & 0.106 \\
Other parenchymal brain lesions & $2(5.4)$ & $1(7.1)$ & 1.000 \\
Sinusitis & $21(56.8)$ & $8(57.1)$ & 0.980 \\
Otitis-mastoiditis & $3(8.1)$ & $1(7.1)$ & 1.000 \\
\hline
\end{tabular}

Data are presented as number (\%).

${ }^{*}$ Chi-square test or Fisher's exact test, where appropriate.

Table 3. Incidence of Cranial Magnetic Resonance Imaging Findings by Disease Activity

\begin{tabular}{lccc}
\hline & Quiescent disease & Moderate to severe disease & p-value \\
\hline All patients (n=51) & 36 & 15 & 0.7485 \\
White matter lesions & $12(33.3)$ & $1(26.7)$ & 1.0000 \\
Other parenchymal brain lesions & $2(5.6)$ & $1(6.7)$ & 0.7657 \\
Sinusitis & $21(58.3)$ & $1(6.7)$ & 1.0000 \\
Otitis-mastoiditis & $3(8.3)$ & 10 & 0.2603 \\
Patients with ulcerative colitis (n=37) & 27 & $2(20)$ & 0.4730 \\
White matter lesions & $12(44.4)$ & $1(10)$ & 0.7165 \\
Other parenchymal brain lesions & $1(3.7)$ & $5(50)$ & 1.0000 \\
Sinusitis & $16(59.3)$ & $1(10)$ & 5 \\
Otitis-mastoiditis & $2(7.4)$ & $5(40)$ & 0.1099 \\
Patients with Crohn's disease (n=14) & 9 & 0 & 1.0000 \\
White matter lesions & 0 & $2(40)$ & 0.5804 \\
Other parenchymal brain lesions & $1(11.1)$ & 0 & 1.0000 \\
Sinusitis & $6(66.6)$ & $1(11.1)$ & \\
Otitis-mastoiditis & & & \\
\hline
\end{tabular}

Data are presented as number (\%). For the comparisons, Fisher's exact test was used. 
ileal and ileocolic involvement, respectively, with 28.6\% having undergone prior surgery due to Crohn's disease (bowel resection or perianal fistulae operation). Overall, mild, moderate or severe disease activity was found in 70.6\%, 23.5\%, and 5.92\% of the IBD patients, respectively.

\section{MRI findings}

Fig. 1 shows an example MRI image of an UC patient with a large white matter lesion. White matter lesions, other brainstem parenchymal lesions $(n=3$, tentorial mass, encephalomalacia, and partial empty sella, respectively), and otitis-mastotidis occurred at a similar frequency in IBD and control patients ( $p>0.05)$, while a diagnosis of sinusitis was made significantly more frequently in IBD patients compared to controls (56.9\% vs 33.3\%, $\mathrm{p}=0.041$ ) (Fig. 2). All subjects with pathological findings on MRI had normal neurological examination.

Among those subjects with white matter lesions, the number of such lesions was significantly higher in IBD patients compared to controls $(12.75 \pm 19.78$ vs $3.20 \pm 2.90, \mathrm{p}<0.05)$. On the other hand, there were no significant differences between UC and Crohn's disease patients with regard to MRI findings (Table 2).

In addition, the incidence of white matter lesions and other brain parenchymal lesions, sinusitis, and otitis-mastotidis did not differ significantly with disease activity ( $p>0.05$ for all) (Table 3).

Among UC patients and controls, those with white matter lesions were significantly older as compared to those without such lesions ( $\mathrm{p}<0.01$, for both). In controls and IBD patients, gender had no effect on the incidence of white matter lesions ( $>>0.05)$.

\section{DISCUSSION}

Few studies have examined neurological and psychiatric complications in IBD, and those examining neurological complications usually consist of sporadic case reports with scarce information on their incidence. IBD has been reported to cause vasculitis in small vessels, and autoimmune mechanisms and vasculitis affecting the small vessels of the central and peripheral nerves have been proposed to play a role in the etiology of neurological manifestations. ${ }^{11}$ Vasculitis of the CNS is a known complication of chronic IBD and pathological vascular changes have been documented by cranial MR angiography (MRA) in these patients. For instance, Schluter et al. ${ }^{12}$ showed typical vessel changes in MRA suggesting angiitis in a female patient with Crohn's disease accompanied by CNS vasculitis, and in a 39-year-old female patient with Crohn's disease, the same authors detected white matter lesions using MRI. Cranial MRA showed narrowing at those locations that was explained by cerebral vasculitis. Another related finding was the observed increase in the risk of stroke in younger Crohn's disease patients in a large case-controlled demographic study conducted by Andersohn et al. ${ }^{13}$ However, pathophysiological mechanisms responsible for the CNS vasculitis associated with IBD are far from being clear.

In the study by Vernooij et al. ${ }^{14}$ in which the coincidental MRI findings in the general population were tested, asymptomatic brain infarctions, cerebral aneurysms, and primary benign tumors (mostly meningiomas) were detected in 7.2\%, 1.8\%, and $1.6 \%$ of the study sample, respectively. Some MR images associated with no symptoms in most healthy individuals may prove to be important disease markers in certain subjects including but not limited to those with multiple sclerosis or other demyelinating diseases, metabolic and toxic conditions, etc. Incidence of focal white matter lesions is increased in patients with systemic vasculitis due to systemic lupus erythrematosus ${ }^{15}$ or Behçet's disease, ${ }^{16}$ and these lesions can be detected by MRI.

Increased number of white matter lesions associated with vascular involvement has been reported in IBD patients undergoing cranial MRI. For example, in five IBD patients with acute neurological deficits, white matter lesions of the CNS have been found with MRI, ${ }^{17}$ and Nemoto et al. ${ }^{18}$ have reported a patient with UC who had sensorineural deafness, brainstem encephalopathy, and white matter lesions. However, studies with asymptomatic patients are relatively scarce. Our literature search revealed only two studies examining the asymptomatic neurological involvement in IBD with cranial MRI. In the first study, Geissler et al. ${ }^{8}$ have found hyperintense focal white matter lesions in $42 \%$ (20/48) of Crohn's disease patients and in 46\% (11/24) of UC patients with no neurological symptoms, while similar lesions existed only in 16\% (8/50) of controls. Hart et al. ${ }^{7}$ searched for white matter lesions in 40 patients with IBD and in 40 controls with tension-type headache and detected such lesions in 12.5\% and 5\% of these two groups, respectively (difference nonsignificant). In this study, 16 (31.4\%) IBD patients had white matter lesions and all had normal neurological examination. No association between these lesions and disease duration, involvement, and activity was found. Of the 30 control subjects, nine (30\%) had white matter lesions. Although the number of patients with white matter lesions was similar in the two groups ( $p>0.05$ ), the number of lesions was significantly higher $(p<0.05)$ in IBD patients. The latter finding is in line with study of Geissler et al., ${ }^{8}$ which showed a similarly increased incidence of white matter lesions in IBD patients. On the other hand, in terms of the percentage of subjects with these lesions in patient and control groups, our study had higher percentages compared to study of Hart et al. ${ }^{7}$ (12.5\% and 5\% vs 31.4\% and 30\%, respectively). This may be attributable to the higher age of patients in the present study. Absence of neurological signs and symptoms in all three studies may be associated with the small size of the lesions or with their clinical insignificance. Similar incidence of white matter lesions in our patient and control groups might be because the control group comprised of patients with headache 
without established neurological origin. Therefore, patients with nonspecific headache may have a tendency to have these lesions when compared to healthy population.

In our study, MRI findings suggestive of sinusitis were significantly more common in IBD patients compared to controls. To our knowledge, such an increase in sinusitis in IBD has not been reported before, but there have been some case reports of nasal involvement in Crohn's disease. For example Ulnick and Perkins ${ }^{19}$ have shown biopsy-proven nasal cavity, supraglottic area, and skin involvement in a 45-year-old male patient with Crohn's disease; Kinnear ${ }^{20}$ have found chronic nasal congestion and inflammatory nasopharyngeal tissue in a 36-yearold female patient with Crohn's disease, and Ernst et al. ${ }^{21}$ have detected polypoid changes with pansinusitis in a 17-year-old male patient with Crohn's disease. The latter patient had histological changes compatible with Crohn's disease in biopsy and responded well to oral steroids. Similarly, Pochon et al. ${ }^{22}$ have reported a 38-year-old male patient with bilateral nasal obstruction and watery nasal discharge who responded to steroids. A possible explanation for increased rate of sinusitis may be due to immunosuppressant use. However, most patients with sinusitis in this study had mild to moderate disease activity, with only one sinusitis patient with severe disease. Since immunosuppressants are usually reserved for patients with severe disease, we do not believe that these agents have an effect on the development of sinusitis in this patient group. However, any such potential causal relation deserves further investigation in appropriately designed studies. Although there is the probability that the increased incidence of sinusitis may be incidental since the significance of the difference was marginal, previous reports still suggest a respiratory mucosal involvement in Crohn's disease, which might be the cause of increased occurrence of sinusitis.

Three of our patients with IBD had parenchymal lesions other than white matter lesions. No such lesions were found in controls. Literature data suggests an increased incidence of extracolonic malignancy in IBD. For example, Ekbom et al. ${ }^{23}$ studied 4,776 patients with IBD who had been followed up for almost 50 years and found an increased incidence of cranial tumors along with increased incidence of other extracolonic tumors in those who had severe UC at the time of diagnosis.

A potential limitation of this study is the small sample size. In addition, inclusion of healthy individuals instead of normal patients attending neurology outpatient clinics would better represent normal population. Nevertheless, our findings provide preliminary evidence on asymptomatic brain lesions in IBD. Future studies with more robust designs and better matching of controls and patients would provide further insight into the issue.

In conclusion, we found no increase in the proportion of subjects with white matter lesions among IBD patients compared to controls and the lesions detected did not seem to pose any clinical significance. However, studies with larger sample size and clinical follow-up of the lesions are warranted to draw firm conclusions.

\section{CONFLICTS OF INTEREST}

No potential conflict of interest relevant to this article was reported.

\section{REFERENCES}

1. Rothfuss KS, Stange EF, Herrlinger KR. Extraintestinal manifestations and complications in inflammatory bowel diseases. World J Gastroenterol 2006;12:4819-4831.

2. Lossos A, River Y, Eliakim A, Steiner I. Neurologic aspects of inflammatory bowel disease. Neurology 1995;45(3 Pt 1):416-421.

3. Benavente L, Morís G. Neurologic disorders associated with inflammatory bowel disease. Eur J Neurol 2011;18:138-143.

4. Zois CD, Katsanos KH, Kosmidou M, Tsianos EV. Neurologic manifestations in inflammatory bowel diseases: current knowledge and novel insights. J Crohns Colitis 2010;4:115-124.

5. Scheid R, Teich N. Neurologic manifestations of ulcerative colitis. Eur J Neurol 2007;14:483-493.

6. Agranoff D, Schon F. Are focal white matter lesions in patients with inflammatory bowel disease linked to multiple sclerosis? Lancet 1995;346:190-191.

7. Hart PE, Gould SR, MacSweeney JE, Clifton A, Schon F. Brain white-matter lesions in inflammatory bowel disease. Lancet 1998;351:1558.

8. Geissler A, Andus T, Roth M, et al. Focal white-matter lesions in brain of patients with inflammatory bowel disease. Lancet 1995;345:897-898

9. Truelove SC, Witts LJ. Cortisone in ulcerative colitis: final report on a therapeutic trial. Br Med J 1955;2:1041-1048.

10. Best WR, Becktel JM, Singleton JW, Kern F Jr. Development of a Crohn's disease activity index. National Cooperative Crohn's Disease Study. Gastroenterology 1976;70:439-444.

11. Jennette JC, Falk RJ. Small-vessel vasculitis. N Engl J Med 1997;337:1512-1523.

12. Schluter A, Krasnianski M, Krivokuca M, Spielmann RP, Neudecker S, Hirsch W. Magnetic resonance angiography in a patient with Crohn's disease associated cerebral vasculitis. Clin Neurol Neurosurg 2004;106:110-113.

13. Andersohn F, Waring M, Garbe E. Risk of ischemic stroke in patients with Crohn's disease: a population-based nested casecontrol study. Inflamm Bowel Dis 2010;16:1387-1392.

14. Vernooij MW, Ikram MA, Tanghe HL, et al. Incidental findings on brain MRI in the general population. N Engl J Med 2007;357:1821-1828.

15. Bell CL, Partington C, Robbins M, Graziano F, Turski P, Kornguth S. Magnetic resonance imaging of central nervous system lesions in patients with lupus erythematosus. Correlation with clinical re- 
mission and antineurofilament and anticardiolipin antibody titers. Arthritis Rheum 1991;34:432-441.

16. Erdem E, Carlier R, Idir AB, et al. Gadolinium-enhanced MRI in central nervous system Behcet's disease. Neuroradiology 1993;35:142-144.

17. de Lau LM, de Vries JM, van der Woude CJ, et al. Acute CNS white matter lesions in patients with inflammatory bowel disease. Inflamm Bowel Dis 2009;15:576-580.

18. Nemoto H, Iguchi H, Ichikawa Y, Wakata N, Kurihara T. Ulcerative colitis presenting as sensorineural deafness, brainstem encephalopathy, and white matter lesions. Neurologist 2004;10:165-168.
19. Ulnick KM, Perkins J. Extraintestinal Crohn's disease: case report and review of the literature. Ear Nose Throat J 2001;80:97-100.

20. Kinnear WJ. Crohn's disease affecting the nasal mucosa. J Otolaryngol 1985;14:399-400.

21. Ernst A, Preyer S, Plauth M, Jenss H. Polypoid pansinusitis in an unusual, extra-intestinal manifestation of Crohn disease. HNO 1993;41:33-36.

22. Pochon N, Dulguerov P, Widgren S. Nasal manifestations of Crohn's disease. Otolaryngol Head Neck Surg 1995;113:813-815.

23. Ekbom A, Helmick C, Zack M, Adami HO. Extracolonic malignancies in inflammatory bowel disease. Cancer 1991;67:2015-2019. 